CARNETS DE Carnets de géographes

GÉOGRAPHES.

\title{
Jardiner les vacants
}

Fabrique, gouvernance et dynamiques sociales des vacants urbains jardinés du nord-est de l'île-de-France

\section{Kaduna-Eve Demailly}

\section{(2) OpenEdition}

\section{Journals}

Édition électronique

URL : http://journals.openedition.org/cdg/324

DOI : $10.4000 /$ cdg.324

ISSN : 2107-7266

Éditeur

UMR 245 - CESSMA

Référence électronique

Kaduna-Eve Demailly, " Jardiner les vacants », Carnets de géographes [En ligne], 8| 2015, mis en ligne le 01 septembre 2015, consulté le 24 septembre 2020. URL : http://journals.openedition.org/cdg/324 ; DOI : https://doi.org/10.4000/cdg.324

Ce document a été généré automatiquement le 24 septembre 2020

\section{(c) $(1) \odot$}

La revue Carnets de géographes est mise à disposition selon les termes de la Licence Creative Commons Attribution - Pas d'Utilisation Commerciale - Pas de Modification 4.0 International. 


\title{
Jardiner les vacants
}

\author{
Fabrique, gouvernance et dynamiques sociales des vacants urbains \\ jardinés du nord-est de l'île-de-France
}

\author{
Kaduna-Eve Demailly
}

1 Apparu à la fin des années 1990 en France, le jardin partagé est décrit comme le nouvel espace vert à la mode manifestant un attachement à l'environnement ainsi qu'un désir de loisir, de convivialité et d'implication citoyenne dans la gestion d'espaces urbains. Son essor dans les villes françaises est largement favorisé par les municipalités qui mettent des terrains à disposition des associations gestionnaires dans le cadre de conventions. Dans l'héritage du jardin familial, le jardin partagé s'inscrit également dans la filiation du community garden nord-américain en ce qu'il traduit notamment le souhait d'un investissement citadin des espaces délaissés métropolitains. Dans le cadre $\mathrm{du}$ développement durable, promouvant une ville compacte, la friche s'envisage désormais comme un support de renouvellement urbain et d'initiatives citoyennes (Chaline, 1999 ; Janin et Andres, 2008).

2 Nous avons alors choisi de nous intéresser à un type spécifique de jardin partagé : le jardin partagé installé sur une friche, que nous avons nommé vacant jardiné institutionnalisé (VJI). Ce dernier se définit comme un espace urbain jardiné par des citadins dans le cadre d'une contractualisation avec le propriétaire, dans l'attente d'une affectation pérenne.

3 Cette thèse vise à comprendre dans quelle mesure le VJI témoigne d'une reconfiguration des modes du «faire» et du «vivre» la ville. Pour une approche globale du fait urbain au prisme du VJI, notre problématique se décompose en deux volets focalisés sur la production de territoires urbains et l'urbanité. Les modalités du «faire » la ville questionnent les dynamiques de coproduction et de gouvernance de ces territoires mais aussi la trajectoire et la valorisation de vacants urbains en jardins partagés. Quant aux modalités du «vivre» la ville, elles renvoient au rôle social, politique et écologique de ces territoires.

4 En croisant la surreprésentation des terrains vacants et la carence en espaces verts, nous avons défini le quart nord-est de l'île de France comme terrain de recherche. En définitive, 48 vacants jardinés (dont 10 ont disparu au cours de cette recherche) ont été 
étudiés : 33 sont situés à Paris, essentiellement dans le nord-est de la commune et 15 sont localisés dans les autres départements étudiés, principalement en Seine-SaintDenis (figure 1).

Figure 1 : les vacants jardinés institutionnalisés étudiés

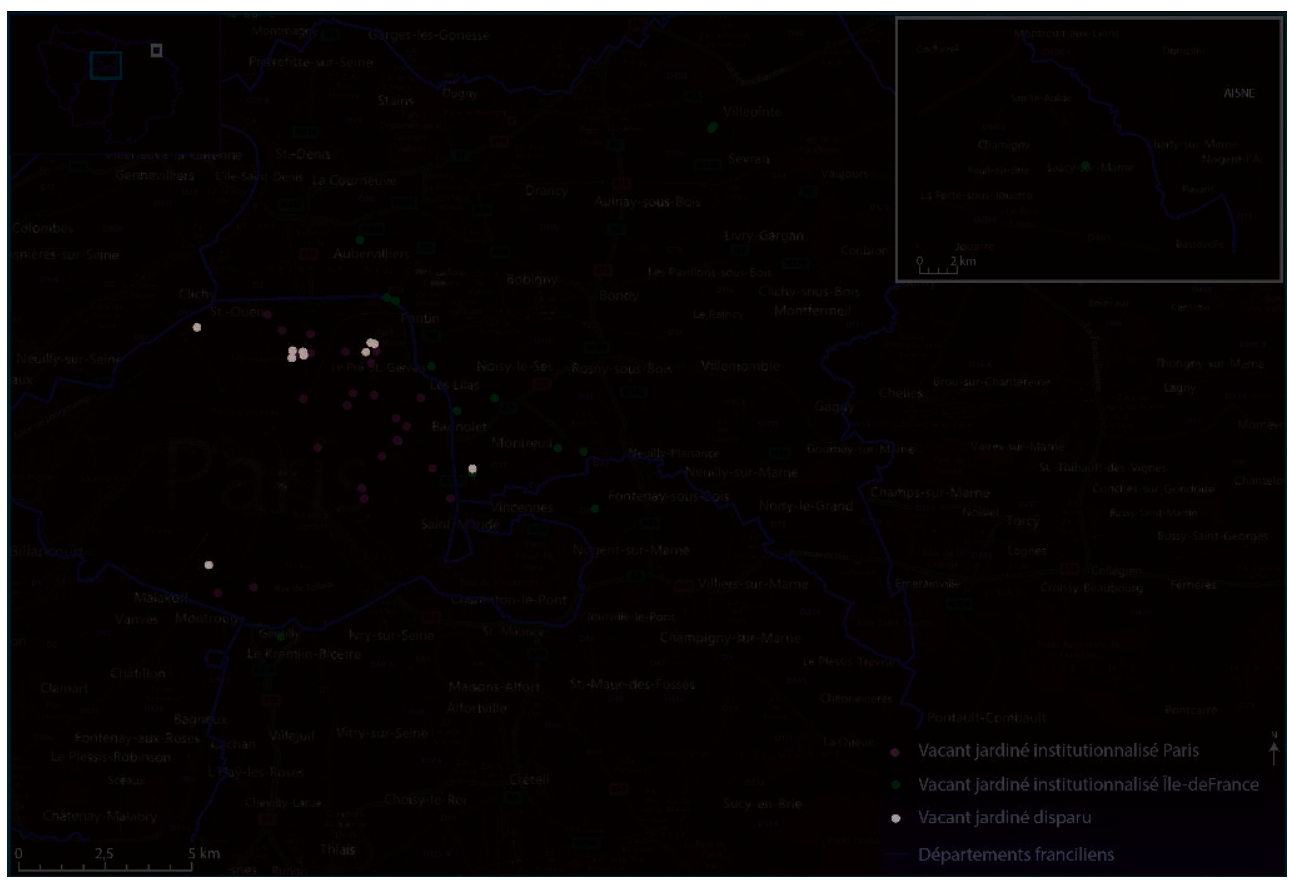

5 Afin de caractériser des discours et des pratiques, l'analyse des documents d'encadrement politique des VJI a été conjuguée à la réalisation d'un matériau propre. Les terrains étudiés ont fait l'objet de phases d'observation (observation simple et une observation participante) tandis que 43 entretiens ont été réalisés auprès d'acteurs institutionnels et d'usagers. Enfin, 130 questionnaires ont été soumis aux adhérents de 16 VJI parisiens. Parallèlement, l'examen de sources quantitatives a permis de préciser les contextes socio-économiques des sites étudiés (données carroyées de l'INSEE) et de spécifier la propriété, les types de vacant et les orientations de valorisation à l'aide du Mode d'Occupation du Sol de l'île-de-France, de l'EVOLUMOS et des données cadastrales.

$6 \quad$ Les principaux apports de cette étude se déclinent en 4 axes : gouvernance, sociabilités et environnement, trajectoires et contextes urbains.

\section{Des territoires coproduits mais une gouvernance inaboutie}

7 L'analyse de la création de ces territoires met à jour leur coproduction associant municipalités, propriétaires, associations et riverains (figure 2). 
Figure 2 : la coproduction des vacants jardinés institutionnalisés

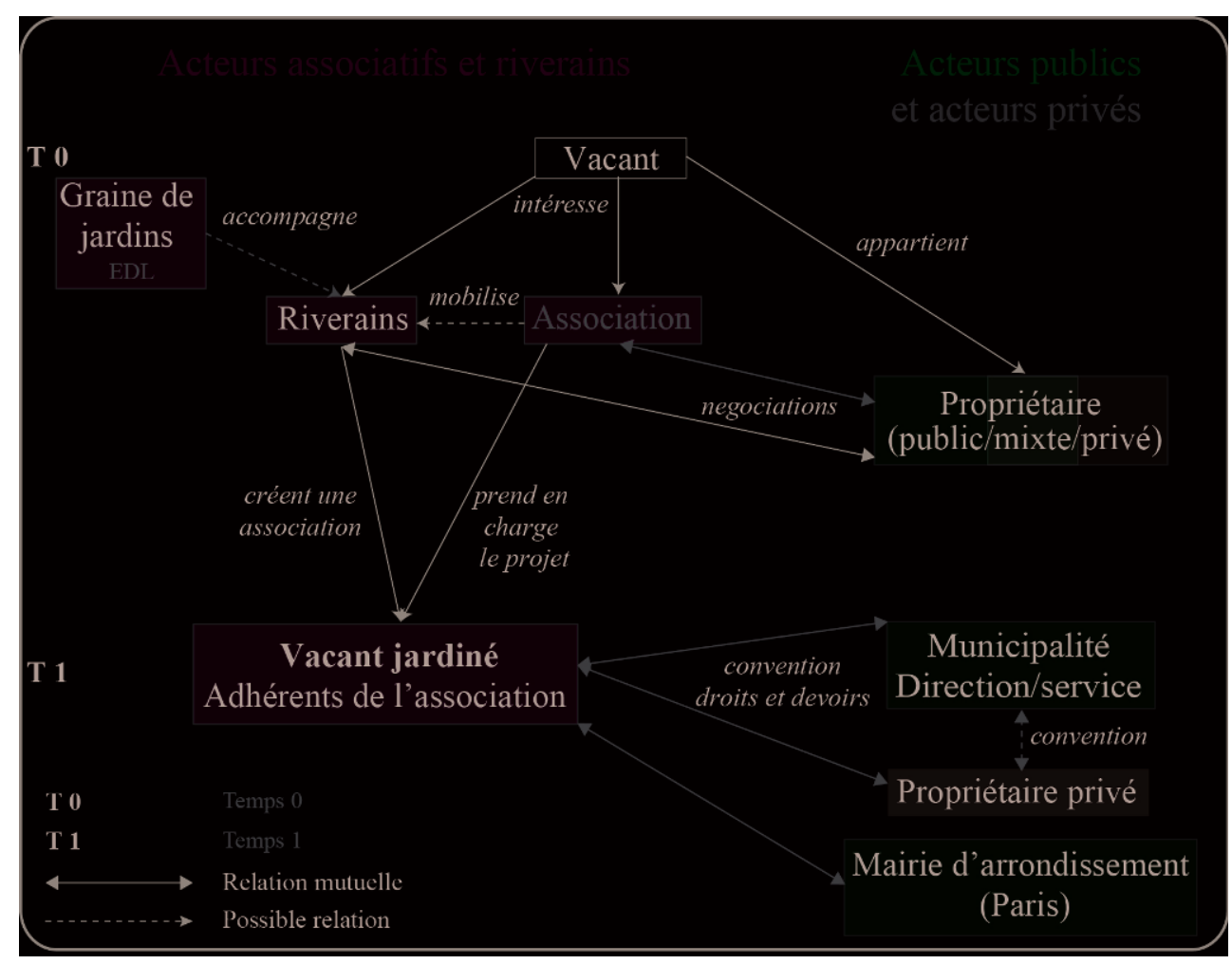

8 Mais si les usagers gèrent et animent les sites au quotidien, leur participation à la décision reste modeste. La municipalité en tant que propriétaire du foncier apparait comme l'acteur déterminant.

\section{Des territoires de sociabilités et de sensibilisation à l'écologie}

9 Malgré l'organisation d'événements, la mise en place de partenariats et de permanences d'ouverture, l'impact du VJI à l'échelle du quartier est relatif ; la création de liens sociaux est circonscrite à l'enceinte du jardin. Ce territoire s'apparente à un «club» au sens de Chris Webster (2002). En outre, en dépit d'une survalorisation du rôle écologique des vacants jardinés, les dispositifs mis en place pour favoriser une gestion écologique du site et la biodiversité apparaissent standardisés (figure 3) et les VJI ne font pas l'objet de protection réglementaire. Ces territoires constituent avant tout des outils de sensibilisation. 


\section{Figure 3 : I'hôtel à insectes : un des dispositifs favorisant la biodiversité les plus répandus}

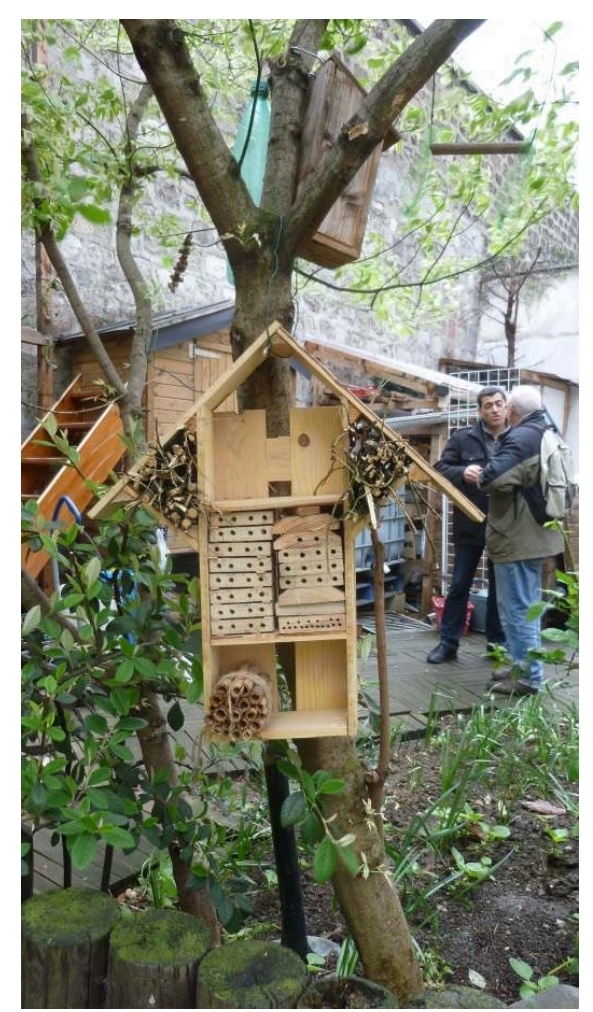

Petits Prés Verts, avril 2011

\section{Des territoires temporaires pour une ville durable}

10 L'affectation de vacants en jardins partagés est envisagée comme une valorisation temporaire de terrains en vue d'une affectation jugée prioritaire comme la construction de logements ou d'équipements. On observe donc une consolidation de l'association entre terrain vacant et jardin, traduisant une rentabilisation accrue des espaces et des rythmes urbains au profit des propriétaires et des municipalités.

\section{Des territoires urbains composites marqués par la discontinuité}

11 L'analyse de la composition socioéconomique des usagers parisiens met en évidence une relative hétérogénéité des profils socioéconomiques. Par contre, la mixité intergénérationnelle est faible (surreprésentation des 40-59 ans) et le sex-ratio déséquilibré (70\% de femmes). A l'échelle urbaine, les VJI se situent sur des zones de « couture ». Les discontinuités relatives à la durée d'occupation des logements des ménages témoignent d'un processus de gentrification à l'œuvre et révèlent une mobilisation du VJI dans le cadre du projet urbain.

Le vacant jardiné institutionnalisé constitue une manifestation de l'injonction contemporaine du développement durable, qui se traduit par des évolutions dans la 
façon de produire et de concevoir la ville au profit des municipalité et des inflexions dans la façon de vivre la ville qu'il est nécessaire de relativiser et d'appréhender à l'échelle micro-locale.

\section{Lien électronique de la thèse}

http://jardinons-ensemble.org/spip.php?article2632

\section{Discipline \\ Géographie}

\section{Directeurs}

Laurent Simon et Renaud Le Goix

\section{Université}

Paris I Panthéon-Sorbonne, UMR 7533 LADYSS

Membres du jury de thèse, soutenue le 4 novembre 2014

Pétros Petsimeris, Professeur Université Paris I (président)

Paul Arnould, Professeur ENS Lyon (rapporteur)

Lise Bourdeau-Lepage, Professeur Université Lyon III (rapporteur)

Raphaël Mathevet, Chargé de recherche CNRS (membre du jury)

Laurent Simon, Professeur Université Paris I (directeur)

Renaud Le Goix, Professeur Université Paris VII (directeur)

Situation professionnelle à l'issue de la thèse

ATER Université Paris-Est Créteil, Chercheur associée au LADYSS

Contact de l'auteur

kadunaeve.demailly[at]gmail.com

\section{BIBLIOGRAPHIE}

CHALINE C. (1999), La régénération urbaine, Paris, Presses Universitaires de France.

JANIN C. et ANDRES L. (2008) « Les friches : espaces en marge ou marges de manœuvre pour l'aménagement des territoires ?", Annales de géographie, nº 663, p. 62-81.

WEBSTER C. (2002) «Property rights and the public realm: gates, green belts, and Gemeinschaft », Environment and Planning B: Planning and Design, vol. 29, n 3, p. 397-412.

\section{INDEX}

Thèmes : Carnets de soutenances 